Bisquerra Alzina, R. \& Filella Guiu, G. (2018). Análisis del vocabulario emocional en el profesorado de lengua. Revista Electrónica Interuniversitaria de Formación del Profesorado, 21(1), 161-172.

DOI: http://dx.doi.org/10.6018/reifop.21.1.298421

\title{
Análisis del vocabulario emocional en el profesorado de lengua
}

Rafael Bisquerra Alzina', Gemma Filella Guiu2

${ }^{1}$ Universidad de Barcelona, ${ }^{2}$ Universidad de Lleida

\section{Resumen}

La escasez de literatura previa que explore y estudie el efecto de la adquisición y desarrollo de un vocabulario emocional adecuado en el entorno escolar se suma al hecho de que no existe una educación emocional sistemática en los centros escolares. A partir de la hipótesis de que el profesorado posee un vocabulario emocional reducido, y que por ende, los alumnos no adquieren el nivel deseado de vocabulario emocional, el presente estudio aporta información sobre este aspecto a partir de un cuestionario aplicado a 276 profesores de lengua y 244 estudiantes de tercer curso del Grado de Primaria. Los resultados muestran que se desconocen de manera relevante las posibilidades y potencial del vocabulario emocional. Esto señala la necesidad de desarrollar un vocabulario emocional más rico tanto en profesores, especialmente de lengua y magisterio, como en los alumnos.

\section{Palabras clave}

Vocabulario emocional; competencias emocionales; educación emocional; consciencia emocional.

\section{Analysis of emotional vocabulary in language teachers}

\section{Abstract}

The scarcity of previous literature that explores and studies the effect of the acquisition and development of an adequate emotional vocabulary in the scholar environment comes alongside the fact that there is no systematic emotional education programs in the scholar centres. Starting from the hypothesis that the teachers have a reduced emotional 
vocabulary, which is linked to the fact that students do not learn the desired level of emotional vocabulary, the present study provides information to prove this assertion, based on a questionnaire applied to 276 language teachers and 244 undergraduate students of the third year of Primary Education. Results show that there is not enough knowledge of the possibilities and potential of the emotional vocabulary. This fact highlights the importance of creating a richer emotional vocabulary both in teachers, particularly in the ones of language and education, and students.

\section{Key words}

Emotional vocabulary; emotional competences; emotional education; emotional awareness.

\section{Introducción}

Comunicar significa utilizar conceptos, generalmente expresados mediante palabras. El vocabulario es el conjunto de las palabras que se utilizan para comunicar. Hay vocabularios específicos para comunicarse sobre temas concretos: matemáticas, gramática, medicina, biología, física, química, derecho, etc. El dominio del vocabulario señala el horizonte de nuestros conocimientos sobre una materia. A mayor riqueza del vocabulario, mayor es el conocimiento.

La capacidad para comunicar emociones es un tipo especial de conocimiento, que se relaciona, no solo con la inteligencia intrapersonal e interpersonal (Gardner, 2001), sino también se contempla en todos los modelos de inteligencia emocional (Bar-On, 1997, Goleman, 1996; Salovey y Mayer, 1990) y las competencias emocionales (Bisquerra y Pérez, 2007). De hecho, la expresión de las emociones ha sido durante décadas un tema de alto interés que guarda relación con el desarrollo del niño/a en todos los aspectos (Chaplin \& Aldao, 2013).

Como la literatura previa indica, es necesario adquirir un buen vocabulario emocional para así poder expresar correctamente los distintos estados de ánimo que se puedan experimentar y así facilitar la comunicación interpersonal y las relaciones sociales (Bisquerra y Filella, 2003; Cabello, Ruiz-Aranda y Fernández-Berrocal, 2010).

La adquisición de un vocabulario emocional rico está justificada eminentemente por el hecho de que el lenguaje emocional es un instrumento para el autoconocimiento y para las relaciones interpersonales profundas (Bisquerra y Filella, 2003; Gallardo y Sáiz, 2016; López, Barba, y Diez, 2013). Es decir, para poder tomar conciencia de las propias emociones y de las emociones de los demás se necesita un vocabulario emocional elaborado y suficientemente extenso para poder describir de manera precisa qué emoción se está desarrollando y así adaptarse al entorno de manera más eficiente (Chaplin y Aldao, 2013).

Hasta el momento presente existen pocos estudios sobre vocabulario emocional. Un trabajo pionero fue el de Ridgeway, Waters y Kuczaj (1985) sobre la adquisición del lenguaje emocional descriptivo, receptivo y productivo entre los 18 meses y los 6 años, el cual señalaba la importancia de iniciar la adquisición de vocabulario emocional durante este periodo. Posteriormente, Joseph y Strain (2003) señalan también la importancia de desarrollar el vocabulario emocional en la infancia y en momentos críticos del desarrollo. A su vez, instrumentos como el British Picture Vocabulary Scale (BPVS) incluye un apartado sobre emociones (Dunn, Whetton y Burley, 1997). No es hasta 2016 que los investigadores 
Takšić y Mohorić (2008; Mohoric, 2016) han elaborado un test de vocabulario emocional en lengua croata que ha sido adaptado al portugués (Costa, Faria y Takšić, 2011).

La percepción e identificación de las emociones requiere de un lenguaje emocional preciso. Cuando se pregunta a alguien “¿Cómo te sientes?", muchas veces la respuesta se limita a un simple "bien" o "mal". A veces escuchamos o decimos: "No sé qué me pasa". Todo esto son ejemplos de las dificultades que tenemos en utilizar un vocabulario emocional apropiado. Cuando una persona es capaz de poner palabra a las emociones, a lo que nos pasa por dentro, estamos en mejores condiciones para gestionar de forma apropiada nuestros conflictos (Cervantes y Gaeta, 2017; Salavera y Usán, 2017). Una persona que experimenta ira muy intensa y no puede poner palabras a lo que le pasa, es más probable que lo exprese con violencia. No queremos extendernos sobre lo obvio que es la importancia y necesidad de desarrollar el vocabulario emocional; este trabajo tiene el objetivo de llamar la atención sobre ello y de proponer que se le preste atención en la educación y en los medios de comunicación.

Esta escasez de literatura coincide con un aumento durante los últimos años de propuestas para mejorar las competencias emocionales en general, pudiéndose afirmar que desde el entorno escolar se utiliza un vocabulario emocional insuficiente y que se beneficiaría notablemente de algún tipo de intervención (Filella, 2014, Filella, Cabello, Pérez- Escoda, y Ros, 2016, Soldevila, Filella, Ribes, y Agulló, 2007). Este hecho repercute indudablemente en limitaciones a la hora de un autoconocimiento, conciencia emocional y regulación emocional, lo cual a su vez, dificulta el desarrollo y adaptación del alumno (Bisquerra, 2014; Gaeta y López, 2013). Así pues, estimular el uso de un lenguaje emocional más rico en la educación sería de un gran beneficio para los estudiantes y también para toda la comunidad.

Por otra parte, la tecnología informática merece una atención especial por su aportación al vocabulario emocional en a partir de herramientas como el Kansei engineering, que en japonés significa "ingeniería emocional" y que se interesa por el desarrollo de productos y servicios a partir de las emociones. Este enfoque se está difundiendo por Japón, Corea y principalmente por Asia, donde la International conference on Kansei engineering and emotion research es un referente. En Europa surge un nuevo campo denominado "emotional design" o "affective engineering". En esta línea se desarrolla, por ejemplo, la investigación para buscar piezas musicales a partir del vocabulario emocional (Zhu y Liu, 2008). Todo este movimiento de ingeniería emocional afecta a la necesidad de un vocabulario cada vez más preciso. Van-Hove, Vanderhoven y Cornillie (2017) proponen el uso de tecnologías como la tablet para el aprendizaje de vocabulario en segundas lenguas, lo cual se podría aplicar al aprendizaje del vocabulario emocional.

De nuevo, este marco plantea la necesidad del desarrollo del vocabulario emocional. Para ello, es importante que los educadores lo dominen y enseñen. El dominio del lenguaje emocional por parte del profesorado es un aspecto clave, especialmente en el profesorado de lengua. Por este motivo, el presente trabajo pretende explorar el dominio del vocabulario emocional por parte del profesorado como requisito para la educación emocional y se propone relacionar el vocabulario con inteligencia emocional.

\section{Método}

\section{Diseño}

En el presente estudio se aplicó una metodología descriptiva cuantitativa basada en la aplicación de un cuestionario con la intención de conocer el dominio del vocabulario 
emocional por parte del profesorado de lengua y estudiantes de tercer curso del Grado de Educación Primaria.

\section{Participantes}

La muestra estuvo constituía, en total, por 520 participantes; 276 profesores de lengua española con una media de años como docentes de 12.43 años, y 244 estudiantes universitarios de tercer curso del grado de educación primaria de la Universidad de Lleida. De los profesores, un total de 26 (9.4\%) eran hombres, mientras que 250 (90.6\%) eran mujeres. En los alumnos, 7 (9.59\% de total de alumnos) eran chicos, mientras que 137 ( $90.51 \%$ del total de alumnos) eran chicas.

La media de edad de los profesores estuvo situada en 44.04 años, con una desviación típica de 9.73. En los estudiantes, la media de edad se situó en 20,53 años, con una desviación típica de 0.98. Así pues, en la muestra de profesores, se observa una clara mayoría del género femenino como es habitual entre los profesionales de la educación. De manera parecida, en los alumnos, existió un desequilibrio notable entre el número de chicos y chicas.

La muestra de profesores se consiguió gracias a la implicación de Difusión, organismo especializado en ELE (Español como Lengua Extranjera): www.difusion.com.

La muestra de alumnos se consiguió a través de la autorización de los profesores universitarios para poder ser evaluados durante unos minutos de las clases habituales.

\section{Instrumentos}

Para la muestra de profesorado, en la recogida de datos se administró un cuestionario con 14 ítems elaborado ad hoc. Los primeros ítems son de identificación personal (género, edad, características profesionales, años de docencia, niveles educativos donde imparten docencia) que se han comentado en el apartado sobre descripción de la muestra de participantes. Más adelante, después de describir el procedimiento, se presentan los resultados de algunas preguntas específicas, las cuales son las más representativas de las obtenidas.

En cuanto a los estudiantes de magisterio, se recogieron los datos demográficos de más relevancia (sexo, edad, nivel educativo) y se les pidió que escribieran el mayor número de emociones que conocieran durante 3 minutos.

\section{Procedimiento}

Para la elaboración del presente trabajo se envió el cuestionario mencionado anteriormente a una muestra de profesorado de lengua española de más de 4000 sujetos para que fuera respondido online. Se obtuvo un total de 276 respuestas. Todas ellas se recogieron durante el mes de febrero de 2017.

Para los estudiantes, se aplicó el cuestionario descrito anteriormente en las aulas durante el mes de marzo de 2017.

\section{Resultados}

El análisis de resultados se llevó a cabo con el programa SPSS 20.

Resultados del profesorado 
A continuación se presentan los resultados referidos al dominio del vocabulario emocional por parte del profesorado de lengua. Las preguntas pertenecientes al cuestionario administrado que se muestran son las más representativas del mismo.

Tabla 1.

Descriptivos del ítem: ¿Cuántas emociones crees que podrías escribir?

\begin{tabular}{|l|l|l|}
\hline & Muestra & Valores inferiores a 100 \\
\hline Media & 52.48 & 25 \\
\hline Mediana & 20 & 20 \\
\hline Moda & 20 & 20 \\
\hline Desviación típica & 139.96 & 16 \\
\hline Valor mínimo & 1 & 1 \\
\hline Valor máximo & 1000 & 70 \\
\hline N total & 276 & 254 \\
\hline
\end{tabular}

Como es muestra en la Tabla 1, la pregunta busca inducir a pensar sobre el número de palabras del vocabulario emocional activo de los sujetos. La media de las respuestas obtenidas fue de más de 52 palabras en la muestra total. Los resultados indican una gran variabilidad en las respuestas, reflejada en la alta desviación típica y en el rango de 1000 (mínimo cero, máximo de 1000). Cuando se analiza con detalle la distribución de frecuencias se puede observar que la mayoría responde menos de 100. En la tabla número 2, se muestra la frecuencia de cada una de las emociones escritas más de 8 veces.

Tabla 2.

Palabras recogidas ordenadas por frecuencia de aparición

\begin{tabular}{|l|l|l|l|l|l|l|l|}
\hline Emoción & N & Emoción & N & Emoción & N & Emoción & N \\
\hline tristeza & 234 & soledad & 32 & placer & 27 & éxtasis & 13 \\
\hline alegría & 193 & culpa & 32 & rencor & 27 & agresividad & 13 \\
\hline miedo & 182 & desilusión & 31 & furia & 26 & repulsión & 12 \\
\hline amor & 148 & deseo & 31 & simpatía & 25 & venganza & 12 \\
\hline felicidad & 133 & paz & 30 & disgusto & 25 & gusto & 12 \\
\hline ira & 128 & cólera & 30 & desprecio & 24 & humillación & 12 \\
\hline sorpresa & 107 & curiosidad & 30 & calma & 24 & desasosiego & 12 \\
\hline rabia & 104 & asombro & 29 & duda & 23 & atracción & 12 \\
\hline odio & 93 & seguridad & 27 & timidez & 22 & piedad & 11 \\
\hline enfado & 86 & interés & 27 & optimismo & 21 & soberbia & 11 \\
\hline asco & 76 & inquietud & 27 & paciencia & 21 & fastidio & 11 \\
\hline angustia & 74 & impaciencia & 27 & horror & 21 & depresión & 11 \\
\hline ansiedad & 72 & placer & 27 & serenidad & 21 & enamoramiento & 11 \\
\hline vergüenza & 67 & rencor & 27 & admiración & 21 & intranquilidad & 10 \\
\hline aburrimiento & 66 & furia & 26 & emoción & 21 & resignación & 10 \\
\hline envidia & 65 & simpatía & 25 & excitación & 20 & impotencia & 10 \\
\hline esperanza & 60 & disgusto & 25 & motivación & 19 & desolación & 10 \\
\hline celos & 60 & desprecio & 24 & indignación & 19 & desinterés & 10 \\
\hline entusiasmo & 59 & calma & 24 & pereza & 19 & afecto & 10 \\
\hline tranquilidad & 57 & duda & 23 & pesimismo & 19 & irritación & 9 \\
\hline pena & 57 & timidez & 22 & confusión & 19 & triste & 9 \\
\hline
\end{tabular}




\begin{tabular}{|l|l|l|l|l|l|l|l|}
\hline satisfacción & 55 & optimismo & 21 & amistad & 19 & vergüenza & 9 \\
\hline frustración & 52 & paciencia & 21 & diversión & 19 & insatisfacción & 9 \\
\hline nostalgia & 51 & horror & 21 & coraje & 19 & contento & 9 \\
\hline pasión & 51 & serenidad & 21 & cansancio & 19 & culpabilidad & 9 \\
\hline enojo & 50 & admiración & 21 & rechazo & 18 & duelo & 9 \\
\hline terror & 49 & emoción & 21 & gozo & 18 & bondad & 9 \\
\hline ternura & 49 & excitación & 20 & estrés & 18 & bienestar & 9 \\
\hline compasión & 49 & motivación & 19 & desconfianza & 18 & alegre & 9 \\
\hline orgullo & 48 & indignación & 19 & gratitud & 17 & sentimiento & 8 \\
\hline ilusión & 47 & pereza & 19 & alivio & 17 & nervioso & 8 \\
\hline melancolía & 46 & pesimismo & 19 & apatía & 17 & risa & 8 \\
\hline dolor & 46 & confusión & 19 & repugnancia & 16 & resentimiento & 8 \\
\hline decepción & 45 & amistad & 19 & júbilo & 16 & valor & 8 \\
\hline alegría & 45 & diversión & 19 & amargura & 16 & sufrimiento & 8 \\
\hline euforia & 44 & coraje & 19 & añoranza & 16 & hastío & 8 \\
\hline empatía & 44 & cansancio & 19 & valentía & 15 & perplejidad & 8 \\
\hline cariño & 42 & rechazo & 18 & susto & 15 & sosiego & 8 \\
\hline inseguridad & 40 & gozo & 18 & antipatía & 15 & desamparo & 8 \\
\hline confianza & 40 & estrés & 18 & lástima & 14 & estupor & 8 \\
\hline preocupación & 39 & desconfianza & 18 & remordimiento & 13 & congoja & 8 \\
\hline temor & 39 & curiosidad & 30 & generosidad & 13 & comprensión & 8 \\
\hline nerviosismo & 37 & asombro & 29 & molestia & 13 & desánimo & 8 \\
\hline desesperación & 37 & seguridad & 27 & solidaridad & 13 & desagrado & 8 \\
\hline indiferencia & 36 & interés & 27 & pavor & 13 & agrado & 8 \\
\hline pánico & 35 & inquietud & 27 & agobio & 13 & & \\
\hline depresión & 34 & impaciencia & 27 & arrepentimiento & 13 & & \\
\hline
\end{tabular}

Además, se observan algunos casos extremos (outliers) que responden 200, 300 y hasta mil. Un análisis pormenorizado de la distribución induce a suprimir los "outliers" para evitar que distorsionen los valores centrales (media). Si se suprimen todas las respuestas superiores a 100 se obtiene la columna "valores inferiores a 100". Lo interesante del cambio es que suprimiendo 22 sujetos (outliers), los resultados son mucho más centrados, con media, mediana y moda entre 20 y 25.

De manera diferente, como se puede apreciar en la tabla 3, en la columna "muestra" se presentan los resultados de las creencias sobre el número total de palabras para denominar emociones en lengua castellana. Aquí tenemos una variabilidad mucho mayor todavía: desviación típica de 2452 y rango de 20000.

Tabla 3.

Descriptivos del ítem: ¿Cuántas palabras para denominar emociones crees que hay en total?

\begin{tabular}{|l|l|r|}
\hline & Muestra & Valores inferiores a 200 \\
\hline Media & 634.86 & 56 \\
\hline Desviación típica & 2452.04 & 37.5 \\
\hline Valor mínimo & 1 & 1 \\
\hline Valor máximo & 20000 & 180 \\
\hline N total & 276 & 211 \\
\hline
\end{tabular}


Analizando la distribución de frecuencias con detalle se observa que hay algunos valores extremos (outliers) que puede que distorsionen hasta cierto punto los estadísticos de tendencia central. Por este motivo, se procedió a eliminar los valores extremos paso a paso hasta lograr una estabilidad con un mínimo de sujetos eliminados. Los resultados de este procedimiento se pueden observar en la columna "valores inferiores a 200". En total, se eliminaron 65 sujetos que son los que han respondido que hay más de 200 emociones.

Además de las anteriores, el cuestionario presenta dos preguntas principales: 1) escribe todas las emociones que conozcas durante tres minutos; 2) continua escribiendo todas las emociones que conozcas controlando el tiempo añadido que va a utilizar. La tabla 4 presenta los resultados. La última columna es el tiempo total invertido. De esta tabla cabe destacar que la media está entre 15 (en 3 minutos) y 25 (con todo el tiempo necesario); el máximo es 45 y 80 respectivamente. En el apartado de discusión se comentan estos resultados.

Los estadísticos del tiempo invertido reflejan que la mayoría en 10 minutos ha escrito todas las emociones que conocen. Solamente algunos llegan a utilizar hasta un máximo de 25 minutos.

Tabla 4.

Descriptivos del total de emociones escritas de la muestra total $(n=276)$.

\begin{tabular}{|l|l|l|l|}
\hline & En 3 minutos & En total & Tiempo \\
\hline Media & 15.17 & 25.05 & 7 \\
\hline Mediana & 13 & 21 & 6 \\
\hline Moda & 9 & 16 & 4 \\
\hline Desv. típica & 8.11 & 14.77 & 3.39 \\
\hline Mínimo & 2 & 2 & 3 \\
\hline Máximo & 45 & 80 & 24 \\
\hline
\end{tabular}

A estos datos se puede añadir que la correlación entre las palabras escritas en 3 minutos y las escritas en el tiempo total utilizado es de 0.822 , lo cual es una correlación muy alta que sugiere que con solo 3 minutos se puede tener una buena estimación del vocabulario emocional de un sujeto.

Las correlaciones entre el número de palabras escritas y la edad o los años de experiencia docente son muy bajas.

Según los resultados obtenidos, del total de 6719 palabras escritas por la muestra de sujetos, se ha obtenido una media por sujeto de 25 palabras. Las emociones principales son repetidas por la mayoría de sujetos. Pero en total se han escrito 1187 palabras distintas. A continuación se presenta unas tablas resumen de los resultados.

\section{Resultados de los estudiantes del Grado de Educación Primaria}

De manera similar a los resultados expuestos en la Tabla 4, los estudiantes universitarios intentaron escribir el mayor número de emociones posible en 3 minutos.

Como se puede observar en estos datos, los estudiantes universitarios del grado de educación primaria no muestran una media tan elevada como lo hacen los profesores estudiados. De hecho, el rango de emociones escrito por los profesores. 
Tabla 5 .

Descriptivos del total de emociones escritas en la

muestra de alumnos universitarios $(n=244)$.

\begin{tabular}{|l|l|}
\hline & En 3 minutos \\
\hline Media & 9.16 \\
\hline Desv. típica & 4.25 \\
\hline Mínimo & 0 \\
\hline Máximo & 24 \\
\hline
\end{tabular}

Tabla 6.

Descriptivos del total de emociones escritas agrupadas en galaxias en la muestra de alumnos universitarios $(n=244)$.

\begin{tabular}{|l|c|c|c|c|c|c|c|c|c|}
\hline & Rabia & Asco & Tristeza & Ansiedad & Alegría & Amor & Felicidad & Sorpresa & Miedo \\
\hline Media & 1.28 & .12 & 1.39 & .33 & .87 & .81 & .72 & .15 & .86 \\
\hline $\begin{array}{l}\text { Desv. } \\
\text { típica }\end{array}$ & 1.33 & .32 & 1.32 & .62 & .88 & .95 & .64 & .45 & .74 \\
\hline Mínimo & 0 & 0 & 0 & 0 & 0 & 0 & 0 & 0 & 0 \\
\hline Máximo & 3 & 1 & 9 & 2 & 3 & 5 & 3 & 1 & 4 \\
\hline
\end{tabular}

Cabe señalar, en el caso de los estudiantes, que, de manera similar a lo que sucede con los profesores, el número de emociones escritas es superior en las emociones principales. Por ejemplo, se mencionan emociones de la familia de la rabia un promedio de 1,28 y de la tristeza un promedio de 1.39, del miedo, un o'86 y de la familia de la alegría se mencionan un o'87, del amor un o' 81 de media.

En la tabla número 7, se muestra la frecuencia de cada una de las emociones escritas más de 8 veces por los estudiantes universitarios en los tres minutos disponibles.

Tabla 7.

Recuento de las emociones más citadas (más de 8 veces) durante tres minutos.

\begin{tabular}{|l|c|l|c|}
\hline \multicolumn{1}{|c|}{ EMOCIÓN } & N & EMOCIÓN & N \\
\hline Tristeza & 165 & llusión & 21 \\
\hline Rabia & 153 & Comprensión & 20 \\
\hline Alegria & 148 & Soledad & 19 \\
\hline Miedo & 147 & Dolor & 17 \\
\hline Felicidad & 97 & Culpa & 17 \\
\hline Amor & 90 & Confianza & 16 \\
\hline Enfado & 71 & Intranquilidad & 14 \\
\hline Frustración & 64 & Anhelo & 14 \\
\hline Empatía & 50 & Estima & 14 \\
\hline Ánimo & 49 & Afecto & 12 \\
\hline Equilibrio & 41 & Optimismo & 12 \\
\hline Serenidad & 37 & Diversión & 12 \\
\hline Euforia & 36 & Pena & 11 \\
\hline Decepción & Solidaridad & 11 \\
\hline Contento & 32 & Violencia & 11 \\
\hline Sorpresa & 32 & Admiración & 10 \\
\hline Angustia & 31 & Satisfacción & 10 \\
\hline Envidia & 30 & Temor & 10 \\
\hline Nerviosismo & 28 & Inseguridad & 10 \\
\hline
\end{tabular}




\begin{tabular}{|l|l|l|c|}
\hline Asco & 26 & Calma & 10 \\
\hline Odio & 25 & Perdón & 9 \\
\hline Preocupación & 25 & Depresión & 9 \\
\hline Remordimiento & 24 & Excitación & 8 \\
\hline Ansiedad & 24 & Desesperación & 8 \\
\hline Impotencia & 23 & Terror & 8 \\
\hline Tranquilidad & 22 & Orgullo & 8 \\
\hline Melancolía & 22 & Placidez & 8 \\
\hline
\end{tabular}

\section{Discusión y conclusiones}

En el presente trabajo se ha pretendido abordar y explorar el vocabulario emocional que actualmente existe entre los profesores de educación formal.

Los resultados muestran en general una gran variabilidad en la media de palabras escritas de los profesores, así como una frecuencia más elevada de determinadas palabras que a su vez, resultan más frecuentes en la vida diaria de los docentes, como podrían ser la tristeza, la alegría y el miedo.

En el caso de los alumnos, aunque también existe una gran variabilidad, las emociones más básicas tienden a mostrar una mayor frecuencia.

Es interesante ver que, en el caso del profesorado, una vez eliminados los valores extremos, la expectativa de palabras que los docentes creen ser capaces de escribir $(\mathrm{N}=25)$ coincide con la que realmente escriben. Este hecho induce a pensar esta podría ser la cantidad de palabras del vocabulario emocional activo de muchas personas, el cual se ha visto que se maneja durante la comunicación interpersonal y la toma de consciencia del mundo afectivo durante la interacción social (Bisquerra y Filella, 2003).

Si nos referimos al vocabulario emocional pasivo, es decir, palabras que existen y que el sujeto puede entender aunque no formen parte del uso habitual, existe una gran diversidad de opiniones. Con toda la muestra se obtiene un máximo de 20000 , que es a todas luces una exageración. A pesar de la escasez de literatura existente, estos datos inducen a pensar que muchas personas, incluso entre el profesorado de lengua, tienen un cierto desconocimiento del volumen del lenguaje emocional, lo cual da lugar a una gran variabilidad.

Si comparamos estos datos con los 567 vocablos del Diccionario de emociones y fenómenos afectivos (Bisquerra y Laymuns, 2016) o los más de 300 del Universo de emociones (Bisquerra, 2015), vemos que el vocabulario emocional activo no llega ni al $10 \%$ de las posibilidades existentes. Este hecho pone de relieve la conveniencia de fomentar el desarrollo del vocabulario emocional en la educación y así potenciar el mundo emocional de los alumnos. En este sentido, sería de vital importancia trabajar y profundizar en primer lugar en el profesorado.

Cuando se analizan las 6719 palabras que se han escrito en total, se puede comprobar que existen 1187 que son distintas entre ellas, lo cual puede ser interpretado como que en conjunto existen muchas palabras relacionadas con las emociones, si bien muchas de ellas no lo son "strictu sensu". Este hecho induce nuevamente a pensar en la conveniencia de una formación sobre vocabulario emocional en el profesorado y en el alumnado. 
Por otro lado, es interesante observar el caso de los estudiantes, ya que aunque se encuentran en un momento de formación profesional que les prepara para ejercer de profesores en un tiempo muy breve, el número de emociones es drásticamente menor al que se obtiene en los profesores. Esto puede explicarse por dos razones: la primera hace referencia a la especialidad de los profesores analizados (lengua). El segundo motivo puede ser el hecho de tener una edad más corta y, por tanto, menos experiencia laboral y vital. Así pues, esto estaría en concordancia con la hipótesis de que, aunque existe cierta escasez de conocimiento del vocabulario emocional, la adquisición de más conocimiento y experiencia vendría acompañado necesariamente, de un aumento del vocabulario emocional. De manera diferente, el número de palabras emocionales distintas utilizadas por el conjunto de la muestra de profesores es muy elevado.

Cabe destacar que aunque en el conjunto de la muestra, el número de palabras escritas es muy elevado, cuando se estudia cada uno de los sujetos en particular, en general no se llega a utilizar el $10 \%$ de posibilidades. De nuevo, se considera que insistir en el desarrollo del vocabulario emocional facilitaría e incrementaría el número de palabras de contenido emocional que el sujeto podría tener en su repertorio.

La ausencia de estudios sobre vocabulario emocional dificulta la comparación de los resultados aquí presentados con otros estudios publicados, aunque sí existen numerosos estudios los cuales destacan la importancia de un adecuado conocimiento de las emociones para un correcto desarrollo del individuo (Di Maggio, Zappulla y Pace, 2016). Además, se ha visto que un mejor autoconocimiento de las emociones de uno mismo ayuda en la adquisición de esta competencia en otros, como es el caso de maestro-alumno (López et al., 2013). Esto sugiere impulsar investigaciones en tal sentido para averiguar y estudiar minuciosamente la implicación del vocabulario emocional. Sería de especial interés además, conocer la evolución del vocabulario emocional a los largo de los años, desde los primeros años de vida hasta la edad adulta, entendiendo la importancia de los periodos críticos del desarrollo y el lenguaje.

Dando un paso más, de cara a las orientaciones didácticas, sería interesante conocer el número de palabras del vocabulario emocional que necesitarían dominar los estudiantes a lo largo del currículum escolar.

Para finalizar, en este estudio exploratorio sobre el vocabulario emocional se han aportado una serie de datos a partir de los cuales se pueden extraer las siguientes conclusiones.

1) El vocabulario emocional reviste una importancia primordial para el autoconocimiento, para la regulación emocional y para unas buenas relaciones interpersonales y sociales.

2) Las nuevas tecnologías introducen la dimensión emocional (Kansei engineering, emotional design, affective engineering) lo cual refuerza la importancia del vocabulario emocional.

3) Hay un extenso vocabulario emocional conocido y utilizado por el conjunto de la población que se puede estimar en unas 500 palabras aproximadamente.

4) Esto contrasta con el limitado uso que se hace del vocabulario emocional que de promedio se sitúa sobre 25 palabras en una muestra de profesorado de lenguaje.

5) Estos datos inducen a fomentar el desarrollo del vocabulario emocional en la educación y en los medios de comunicación.

6) Para eso es necesario la formación del profesorado en educación emocional.

7) El profesorado de lengua debería ser un sector prioritario de formación en vocabulario emocional para poder contribuir a su adquisición por parte del alumnado. 
Los estadísticos del tiempo invertido reflejan que la mayoría en 10 minutos ha escrito todas las emociones que conocen. Solamente algunos llegan a utilizar hasta un máximo de 25 minutos. Todo esto son datos tener en cuenta en futuras pruebas de vocabulario emocional.

\section{Referencias}

Bar-On, R. (1997).The Emotional Intelligence Inventory (EQI): Technical manual.Toronto, Canada: Multi-Health Systems

Bisquerra, R. (2015). Universo de emociones. Valencia: PalauGea.

Bisquerra, R., y Filella, G., (2003). Educación emocional y medios de comunicación. Guidance and mass-media. Comunicar. Revista Científica de Comunicación y Educación, 20, 6367.

Bisquerra, R. (Coord.).(2014). Prevención del acoso escolar con Educación emocional. Bilbao: Desclee de Brouwer

Bisquerra, R., y Laymuns, G. (2016). Diccionario de emociones y fenómenos afectivos. Valencia: PalauGea.

Bisquerra, R., y Pérez Escoda, N. (2007). Las competencias emocionales. Educación XX1, 10, 61-82.

Cabello, R., Ruiz-Aranda, D., y Fernández-Berrocal, P. (2010). Docentes emocionalmente inteligentes. Revista Electrónica Interuniversitaria de formación del profesorado (REIFOP), 13, 1. Enlace web: http://www.aufop.com - Consultada en fecha 25-02-17.

Cervantes, M. C. M., y González, M. L. G. (2017). Desarrollo de competencias emocionales en pre-adolescentes: el papel de padres y docentes. Revista Electrónica Interuniversitaria de Formación del Profesorado, 20(2), 221-235.

Chaplin, T. M., y Aldao, A. (2013). Gender differences in emotion expression in children: A meta-analytic review. Psychological Bulletin, 139(4), 735-765.

Costa, A., Faria, L., y Takšić, V. (2011). Vocabulary of Emotions Test (VET): A Cross-cultural Validation in Portuguese and Croatian Contexts. Psychological Topics, 20, 3, 413-424.

Di Maggio, R., Zappulla, C., y Pace, U. (2016). The Relationship Between Emotion Knowledge, Emotion Regulation and Adjustment in Preschoolers: A Mediation Model. Journal of Child and Family Studies, 25(8), 2626-2635.

Dunn, L. M., Whetton, C., y Burley, J. (1997). The British Picture Vocabulary Scale (2nd ed.). Windsor: NFER-Nelson.

Gaeta, M. L., y López, C. (2013). Competencias emocionales y rendimiento académico en estudiantes universitarios. Revista Electrónica Interuniversitaria de Formación del Profesorado, 16(2), 13-25.

Filella, G. (2014). Aprendre a conviure. Videojuego para el desarrollo de las competencias emocionales. Barcelona. Barcanova.

Filella, G. Cabello, E. Pérez-Escoda, N. y Ros, A. (2016). Evaluation of the Emotional Education pro-gram "Happy 8-12" for the assertive resolution of conflicts among peers. Electronic Journal of Research in Educational Psychology, 14 (3), 582-601.

Gallardo, I. M., y Sáiz, H. (2016). Emociones y actos comunicativos desde la dramatización de situaciones cotidianas. Una propuesta de intervención en Educación Primaria. Revista Electrónica Interuniversitaria de Formación del Profesorado, 19(3), 219-229.

Gardner, H. (2001). La inteligencia reformulada. Las inteligencias múltiples en el siglo XXI. Barcelona: Paidós.

Goleman, D. (1996). Inteligencia emocional. Barcelona: Kairós. 
Joseph, G. E., y Strain, Ph. (2003). Enhancing Emotional Vocabulary in Young Children. Young Exceptional Children, 6, 4, 18-26.

López, A. M. M., Barba, Á. V., y Diez, S. G. (2013). El registro de la emoción en el cuerpo, autoconocimiento y formación del profesorado. Revista Electrónica Interuniversitaria de Formación del profesorado, 16(2), 135-145.

Mohoric, T. (2016). Konstrukcija i validacija Testa razumijevanja emocija u okviru Rosemanova modela emocija. Psychological Topics, 25(2), 223-243.

Ridgeway, D., Waters, E., y Kuczaj, S. A. (1985). Acquisition of emotion descriptive language: Receptive and productive vocabulary norms for ages 18 months to 6 years. Developmental Psychology, 21, 901-908.

Salavera, C., y Usán, P. (2017). Repercusión de las estrategias de afrontamiento de estrés en la felicidad de los alumnos de Secundaria. Revista Electrónica Interuniversitaria de Formación del Profesorado, 20(3), 65-77.

Salovey, P., y Mayer, J. D. (1990). Emotional Intelligence. Imagination, Cognition, and Personality, 9, 185-211.

Soldevila, A., Filella, G., Ribes, R., y Agulló, M. J. (2007). Una propuesta de contenidos para desarrollar la conciencia y la regulación emocional en la Educación Primaria. Cultura y educación, 19(1), 47-59.

Takšić, V., y Mohorić, T. (2008). Vocabulary of Emotions Test (VET): Ability measure of emotional intelligence. Paper presented on XXIX International Congress of Psychology, Berlin, Germany.

Van-Hove, S., Vanderhoven, E., Cornillie, F. (2017). La tablet para el aprendizaje de vocabulario en segundas lenguas: teclado, lápiz digital u opción múltiple. The tablet for Second Language Vocabulary Learning: Keyboard, Stylus or Multiple Choice Comunicar. Revista Científica de Comunicación y Educación, XXV, 50, 1, 53-63.

Zhu, B., y Liu, T. (2008). Research on Emotional Vocabulary-Driven Personalized Music Retrieval. En Pan, Z., Zhang, X., El Rhabili, A., Woo, W., y Li, Y. (Eds.), Technologies for E-Learning and Digital Entertainment. Third International Conference, Edutainment 2008 Nanjing, China, June 25-27, 2008 Proceedings (252-261). Heildelberg: Springer. 